\title{
Aqueous, Glutinous, Cavitary: Primary Effusion Lymphoma
}

\author{
Anubha Bajaj* \\ Department of Pathology, Punjab University, India \\ *Corresponding author: Anubha Bajaj, Histopathologist, Punjab University, India \\ submission: September 12, 2018; Published: September 24, 2018
}

\section{Preface}

Primary effusion lymphoma (formerly known as body cavity lymphoma), is an infrequent, aggressive B cell non-Hodgkin's lymphoma. The human herpes virus 8 (HHV8) or the Kaposi's sarcoma associated herpes virus (KSHV) may be the potential determinant of the malignancy [1]. The viral genesis was determined in conjunction with the Kaposi's sarcoma secondary to the human immune deficiency (HIV) virus and autoimmune deficiency syndrome (AIDS) in 1994 and to the non-Hodgkin's lymphoma in 1995 [1,2]. Subsequently, the world health organization designated the primary effusion lymphoma as a singular, independent neoplasm in 2001 [3]. An estimated $4 \%$ of the HIV related non-Hodgkin's lymphoma and $1 \%$ of the non-viral- HIV related non-Hodgkin's lymphoma may be constituted by PEL [4].

The predominantly male lymphoma (Male to Female ratio is 6:1) may classically appear in the middle aged to elderly, immune compromised individuals with underlying hepatic cirrhosis, recipients of solid organ transplants, patients infected with HIV or those individuals residing in HIV endemic regions $[5,6]$. The majority (6090\%) of PEL cases have a co-existing Epstein Barr viral (EBV) infection, but the role of EBV in the pathogenesis of PEL has not been fully elucidated [7]. The geriatric, HIV non-reactive (HIV-) patients are characteristically non-reactive to the Epstein Barr virus (EBV-) and may appear in the HHV8 endemic regions $[3,8]$. The majority of individuals may have a co-existing AIDS or Kaposi's sarcoma [9]. The pleural, pericardial or peritoneal cavity may be implicated by the disorder [10].
PEL is characterized by a solitary, unilateral and homogenous effusion with the absence of a clinical or a radiographic manifestation of an apparent tumefaction or an extra-cavitary cellular aggregate. On computerized tomography (CT), PEL may lack characteristic or diagnostic features. A recognizable effusion in a severely immune compromised individual or co-existing with an autoimmune deficiency syndrome (AIDS) may be considered PEL [1,2].

\section{Disease Evolution}

The gamma human herpes virus 8 (yHHV 8) may be concordant with various malignancies such as PEL, Kaposi's sarcoma, multi-centric Castleman' Disease, HHV8 related diffuse large B cell lymphoma (DLBCL) and germinotropic lympho-proliferative disease $[4,11]$. The B lymphocyte may be infected by HHV8 in the latent phase and viral replication may arise in the lytic phase, thus initiating the malignant conversion of PEL $[1,8]$. The latent phase of the yHHV8 infection may develop into malignancy by virtue of stimulating the viral transcripts (Table 1). The latency associated nuclear antigen (LANA), viral FLICE inhibitory protein (v FLIP) and viral cyclin (y Cyclin) may be involved in the evolution of HHV8 induced malignancies [1]. The latent phase of the virus may be corroborated by the LANA with the inhibition of the tumour suppressor protein $\mathrm{p}^{53}$ and the retinoblastoma protein, the dys-regulation of the NOTCH pathway, in addition to the cellular development, evolution and survival of the tumour cells [10].

Table 1: Pathological distinction of primary effusion lymphomas from aggressive lymphomas.

\begin{tabular}{|c|c|c|c|c|}
\hline & DLBCL (Immunoblastic) & PBL & ALCL & PEL \\
\hline Morphology & $\begin{array}{l}\text { Diffuse sheets of large } \\
\text { cells, prominent nucleoli, } \\
\text { abundant cytoplasm and } \\
\text { plasmacytoid features }\end{array}$ & $\begin{array}{l}\text { Diffuse cells with abundant } \\
\text { cytoplasm with eccentric } \\
\text { nuclei and smaller nucleoli } \\
\text { resembling plasma cells }\end{array}$ & $\begin{array}{l}\text { Pleomorphic nuclei } \\
\text { with multiple (or single) } \\
\text { prominent nucleoli with } \\
\text { abundant cytoplasm }\end{array}$ & $\begin{array}{c}\text { Variable morphology with } \\
\text { immunoblastic, plasmablas- } \\
\text { tic or anaplastic large cell } \\
\text { lymphoma }\end{array}$ \\
\hline EBV reaction & $90 \%-100 \%$ & $>50 \%$ & Negative & $60 \%-90 \%$ \\
\hline HHV8 reaction & - & - & - & $100 \%$ \\
\hline Immune Phenotype & BCL6-, CD138+, MUM 1+ & $\mathrm{CD} 138+, \mathrm{CD} 38+, \mathrm{CD} 20+$ & $\begin{array}{l}\text { CD30+, EMA+, CD4+, CD2+, } \\
\text { TIA, Granzyme OR Perforin+ }\end{array}$ & $\begin{array}{c}\mathrm{CD} 30+, \mathrm{CD} 38+, \mathrm{CD} 138+, \\
\text { CD45+. }\end{array}$ \\
\hline Cellular Genesis & $\begin{array}{l}\text { Germinal centre or post } \\
\text { germinal centre B cells }\end{array}$ & Post germinal centre cells & Primitive $\mathrm{T}$ cell origin & $\begin{array}{l}\text { Germinal centre (CD10+ or } \\
\text { BCL6+ \& MUM1-) or post } \\
\text { germinal centre B cells }\end{array}$ \\
\hline
\end{tabular}


v Cyclin and v FLIP may enhance the tumour progression by selectively activating the cyclin dependent kinase 6 and the transcription factor nuclear factor kappa B(NF-KB) network, respectively. Multiplication of tumour cells with prohibition of apoptosis may be achieved with the conservation of viral latency. y HHV8 may assemble quantities of viral interleukin 6 (vIL6) which may activate the vascular endothelial growth factor (VEGF) to augment the vascular permeability and increase the incidence of PEL [9]. vIL6 may inhibit apoptosis by repressing the pro-apoptotic cathepsin D (Figure 1).

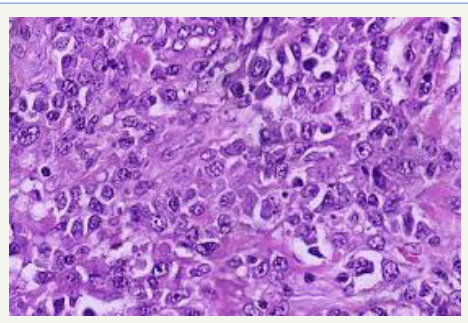

Figure 1: PEL: large cells with nuclear pleomorphism.

Accessory genes of the yHHV8 within the latent viral phase may initiate the malignant transformation through cellular multiplication, cellular binding, apoptosis, angiogenesis, cytokine formulation and propagation of B lymphocytes, thus augmenting the development of the tumour $[4,11]$. The lytic and reproductive stage of HHV8 infection may produce cellular dissolution and demise of the infected cell. Thus, it may be advantageous for the virus to persist in the latent phase in order to augment disease progression and cellular extinction.

\section{Disease Demonstration}

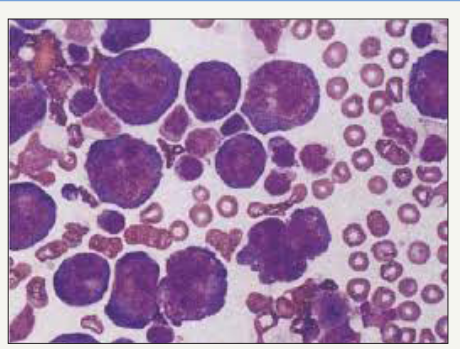

Figure 2: PEL: secondary to KSHV infection: immunoblastic appearance.

Lymphoma induced effusion in the body cavities (pleural, peritoneal, pericardial) may be clinically suspicious for malignancy. The magnitude of the effusion and the underlying disease process may define the clinical aspects of the lymphoma (Figure 2). An immune compromised host who is symptomatic (i.e. short of breath), may have a pleural effusion detectable on imaging, amplification of the abdominal girth and elevation in intra-abdominal pressure or pedal oedema. Such manifestations may indicate cavity specific fluid aggregation due to PEL. Cardiac tamponade accompanied by dizziness, hypotension electrocardiogram (ECG)changes may signify a pericardial involvement. B symptoms such as fever, > 10\% total body weight loss within the preceding six months and drenching night sweats may appear. An extra-cavitary mass may be discerned
(Figure 3). Viscera abutting a body cavity, regional lymph nodes, bone marrow, skin, central nervous system and gastro-intestinal tract may be incriminated by the extension of the disease process $[12,13]$.

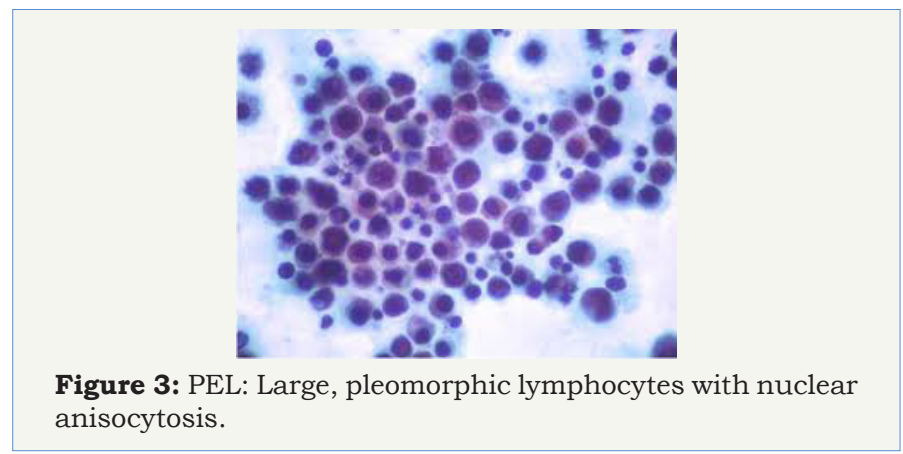

\section{Disease Investigations: Histo-Morphology}

The initial, presenting effusion may be analysed on routine cytology or histopathology. The world health organization elucidates the tumour (PEL) cells as displaying features betwixt the large cell immunoblastic lymphoma or a plasmablastic lymphoma or an anaplastic large cell lymphoma [3]. The enlarged cells delineate a modest to abundant deeply basophilic cytoplasm, mammoth round to irregular nuclei with conspicuous nucleoli (Figure 4). The cells are immune reactive to the lymphoid marker CD45+ although may depict a "null lymphocyte" phenotype in the absence of characteristic $\mathrm{B}$ or T lymphocyte immune phenotypes. Cellular reactions may be inconclusive by immune histochemistry.

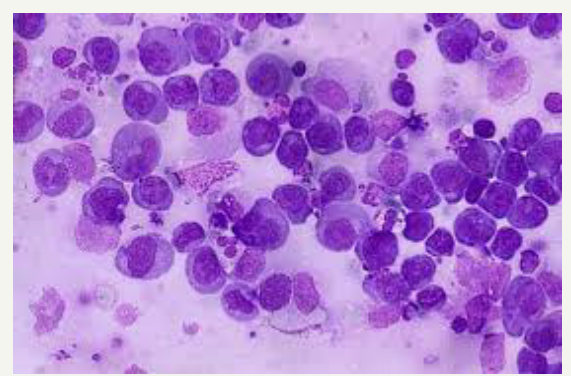

Figure 4: PEL: plasmablastic cells with basophilic cytoplasm.

Majority $(70 \%)$ of the classical Hodgkin's lymphoma exhibit CD30 + reactivity with a lack of CD15- and an abundance of Reed Sternberg cells. Immune markers of plasma cell differentiation such as CD38+ and CD138+ may be exemplified, though a plasma cell myeloma may classically lack a CD45- response [1]. The epithelial membrane antigen (EMA) or the initiating molecules of human leukocyte antigen (HLA-DR) may be inconsistently expressed. The categorical detection of yHHV8 within the nuclei of the tumour cells is diagnostic of the primary effusion lymphoma (Figure 5). The immune histochemical depiction of the latency associated nuclear antigen 1 (LANA1) or the extraction and amplification of the gamma herpes 8 viral de-oxy ribonucleic acid (DNA) by the polymerase chain reaction (PCR)may be a confirmative methodology [1]. The "null phenotype" primary effusion lymphoma may thus be distinguished from lymphomas with identical histology and clinical aspects. 


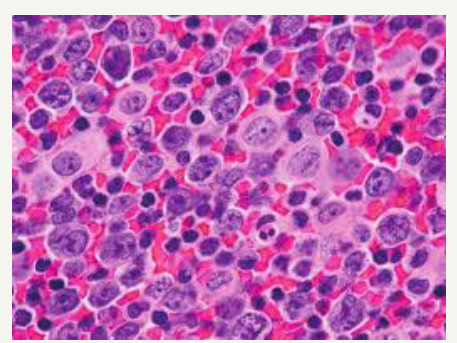

Figure 5: PEL: HHV8 infection, large cells with prominent nucleol.

\section{Genetic Modifications}

The post germinal centre late differentiating B lymphocytes (activated B cells CD10-, MUM 1+, BCL6+/-) of possible plasmablastic origin may be the determinants of the malignant cells in primary effusion lymphoma, as evaluated by the gene expression profiling (GEP) (Table 2). The heavy immunoglobulin (IgG) gene may elucidate clone specific rearrangements which distinguishes the

Table 2: Modified ann arbor staging for non-Hodgkin's lymphoma.

\begin{tabular}{|c|c|}
\hline Stage & Features \\
\hline I & Involvement of a single lymph node region or a lymphatic organ (spleen or thymus or Waldeyer ring) \\
\hline II & Involvement of two or more lymph node regions on the same side of the diaphragm \\
\hline III & Involvement of lymph node regions/structures on both side of the diaphragm \\
\hline IV & Involvement of the extra-nodal sites beyond the designated E. \\
\hline E (for stages I to III) & Involvement of the single extra-nodal site contiguous or proximal to the known nodal site \\
\hline A or B & Absence(A) or Presence (B) of fever, drenching sweats or loss of $>10 \%$ body weight in the preceding six months \\
\hline
\end{tabular}

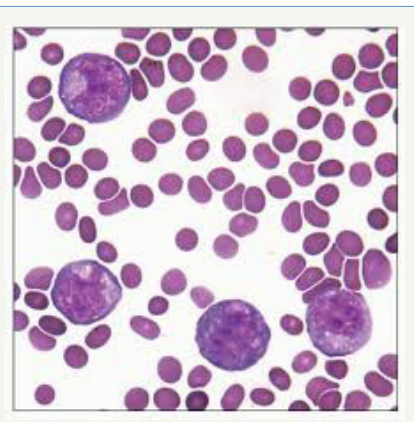

Figure 7: PEL: Enormous cells with chromatin dispersal.

A frequent substitute mutation of BCL6 5' non-coding region nucleotide may be demonstrated in the lymphoma. BCL6 5' mutation essentially exemplifies the metamorphosis of B lymphocytes within its progression through the germinal centre. The characteristic mutation may specifically cite the post germinal centre B lymphocyte as an antecedent of the lymphoma [1]. A common complete or partial trisomy 12 with atypical bands of 1q21-25 may be delineated. Interleukin receptor associated kinase 1 (IRAK1) along with the binding collaboration with MYD 88 may produce a toll like receptor signalling mechanism in order to revive yHHV8. This may extend the survival of PEL cells in culture (Figure 7). The $\mathrm{X}$ chromosome targeted sequencing of PEL may discern the constitutive phosphorylation (mutation) within the lymphoma cells by the interleukin receptor associated kinase 1 (IRAK 1) which may be a
B lymphocyte derivation of the tumour (Figure 6). A reoccurring cytogenetic anomaly or a characteristic mutation for the lymphoma cells has not been ascertained [1]. The classic, multiple genetic reorganizations associated with a non-Hodgkin 's lymphoma or mutations of BCL2, c MYC and TP53 may be absent in the primary effusion lymphoma.

Figure 6: PEL: Immunoblastic appearance with basophilic
cytoplasm.




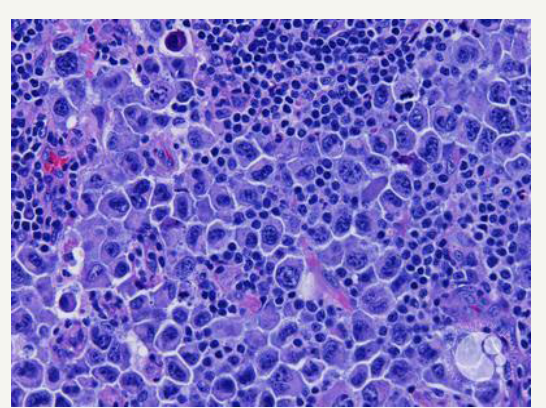

Figure 8: PEL: Mammoth cells with cellular variation.

\section{Therapeutic Options}

As the disease lacks frequency, a quantifiable, therapeutic randomized control trial may be absent. The preferred chemotherapy for primary effusion lymphoma with co-existing HIV infection may be a dose adjusted etoposide, prednisone, vincristine, cyclophophamide and doxorubicin (DA EPOCH) or a cyclophosphamide, doxorubicin, vincristine, prednisone (CHOP) regimen. With the dose adjusted (DA EPOCH) regimen the estimated objective response rate (ORR) may be $87 \%$, the complete response (CR) at $74 \%$ with an approximate four-year disease-free survival (DFS) at $92 \%$ and an overall survival $(\mathrm{OS})$ at $60 \%[1,2]$.

Lympho proliferative malignancies with concordant human immune deficiency virus (HIV+) infection may benefit maximally with anti-retroviral therapy. A worse performance status with a reduced overall survival (OS) may be witnessed in the HIV+ patients lacking therapy (Figure 9). The administration of combined cyclophosphamide, doxorubicin, vincristine, prednisone (CHOP) in the absence of appropriate and concomitant anti-retroviral therapy may display a reduced overall survival (OS) of three months with a lack of complete remission (CR). Apoptosis of the tumour (PEL) cells may manifest through the repression of NF KB pathway, stimulated by the protease inhibitor Lopinavir. Drugs such as Azidothymidine may activate the lymphoma (PEL) cells along with the virus (KSHV) specific CD4+ T cells in order to prohibit the cellular proliferation of the lymphoma [1].

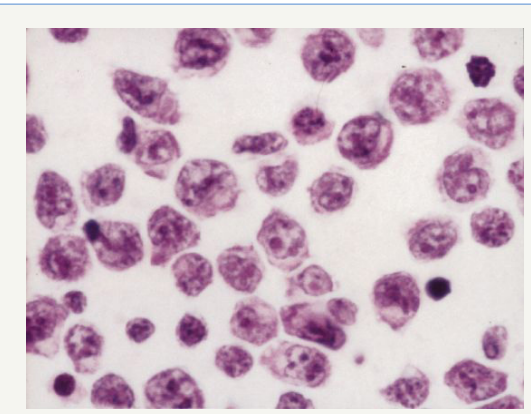

Figure 9: PEL HHV8 induced pleural effusion with anaplastic cells.

\section{Refractory Instances}

The disorder may reoccur within 6 to 8 months in the majority of the patients. The occurrence of co-morbid conditions, the per- formance status (PS) and the achievable therapeutic targets may define the protocol of the treatment.

An amplified chemotherapeutic regimen with a subsequent autologous stem cell transplantation (ASCT) may be suitable in patients lacking the co existent viral infection (HIV-). The instances of PEL with concordant viral (HIV+) infection may benefit from an allogeneic stem cell transplant. Radiation therapy may be insufficient if employed singularly for achieving a complete remission (CR), though may be beneficial for reducing the magnitude of the disease. Radiation therapy may also be applicable for consolidated constituents of the primary effusion lymphoma, accommodated within a single radiation field [2].

Antiviral therapies may be applicable for the viral lysis. Viral multiplication may be responsive to ganciclovir, foscarnet, cidofovir along with a resistance to acyclovir [1]. Talc pleurodesis may be advantageous in the PEL within the body cavities. The visceral and parietal pleural layers may amalgamate to prevent the accumulation of fluid. The sclerosant effect of talc may exhibit an apoptotic influence on the malignant cells. Targeted therapies may include specific proteosomal inhibitors.

Proteosomal activity may be necessitated for the perpetuation of PEL cells and for the viral (KSHV/HHV8) multiplication [14]. A proteosomal inhibitor, Bortezomib may be ineffective when singularly adopted for refractory patients. The proteosomal inhibitor, Bortezomib may be beneficial in combination with PEGlyated liposomal doxorubicin and rituximab, thereby inducing a suitable complete remission (CR). Bortezomib along with a histone deacetylase inhibitor vorinostat may manifest a lytic replication of the virus (KSHV/HHV8) accompanied by a cellular mortality $[1,8,14]$. Brentuximab vedotin (BV) is an antibody drug conjugate against the CD30 molecule (Figure 10). As PEL is immune reactive to the CD30+ molecule, the drug conjugate (BV) may be efficacious in constraining the gap 2 (G2) of the inter-phase of the cell division. The actual efficacy of the molecule may presently be debatable.

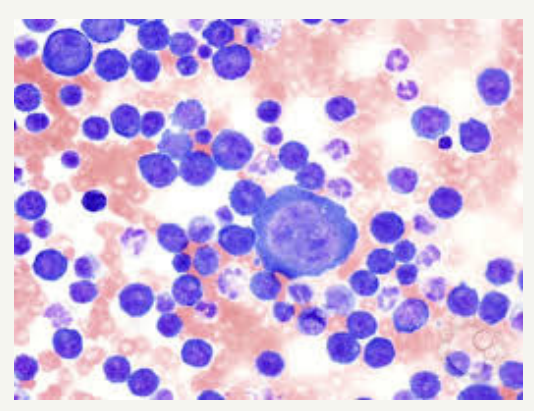

Figure 10: PEL: Immunoblastic tumor cells with cytoplasmic basophilia.

\section{References}

1. National Statistical Institute of Bulgaria. Bulgaria.

2. Buciuniene I, Blazeviciene A, Bliudziute E (2005) Health care reform and job satisfaction of primary health care physicians in Lithuania. BMC Fam Pract 6(1): 10. 
3. Goetz K, Campbell S, Broge B, Brodowski M, Steinhaeuser J, et al. (2013) Job satisfaction of practice assistants in general practice in Germany: an observational study. Family Practice 30(4): 411-417.

4. Linzer M, Baier ML, Williams ES, Bobula JA, Brown RL, et al. (2009) Working conditions in primary care: physician reactions and care quality. Ann Int Med 151(1): 28-36.

5. Wallace JE, Lemaire JB, Ghali WA (2009) Physician wellness: a missing quality indicator. Lancet 374(9702): 1714-1721.

6. Szecsenyi J, Goetz K, Campbell S, Broge B, Reuschenbach B, et al. (2011) Is the job satisfaction of primary care team members associated with patient satisfaction? BMJ Qual Saf 20(6): 508-514.

7. Dowd BE, Kralewski JE, Kaissu AA, Irrgang SJ (2009) Is patient satisfaction influenced by the intensity of medical resource use by their physicians? Am J Manag Care 15(5): 16-21.

8. Engels Y, Dautzenberg M, Campbell S (2006) Testing a European set of indicators for the evaluation of the management of primary care practices. Fam Pract 23(1): 137-147.
9. Van Ham I, Verhoeven AAH, Groenier KH, Groothoff JW, De Haan J (2006) Job satisfaction among general practitioners: a systematic literature review. Eur J Gen Pract 12(4): 174-180.

10. Goetz K, Campbell S, Steinhaeuser J, Joachim S, Sara W, et al. (2011) Evaluation of job satisfaction of practice staff and general practitioners: an exploratory study. BMC Fam Pract 12: 137.

11. Goetz K, Marx M, Marx I (2015) Working atmosphere and job satisfaction of health care staff in kenya: an exploratory study. BioMed Research International p. 7.

12. Heritage B, Pollock C, Roberts L (2015) Confirmatory factor analysis of warr, cook and wall's (1979) job satisfaction scale. Australian Psychologist 50(2): 122-129.

13. Al Eisa I, Al Mutar M, Al Abduljalil H (2005) Job satisfaction of primary health care physicians at capital health region, kuwait. Middle East Journal of Family Medicine 3(3): 1-5.
Creative Commons Attribution 4.0 International License

For possible submissions Click Here

\section{Submit Article}

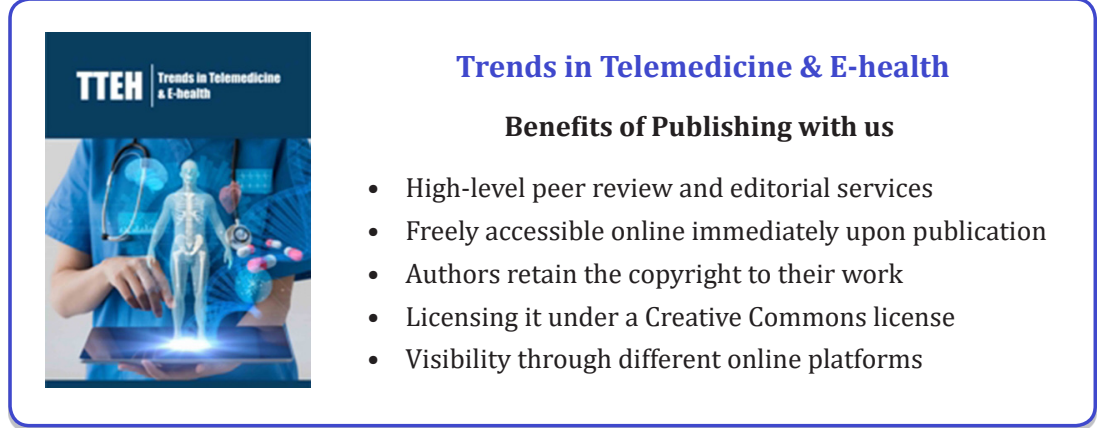

